\title{
Espelho, espelho meu: um estudo sobre autoimagem corporal de estudantes universitários
}

\author{
Mirror, mirror on the wall: a study on body \\ image of university students
}

Rev Bras Ativ Fis Saúde p. 112-120

DOI: http://dx.doi.org/10.12820/2317-

1634.2013v18n1p112

1 Escola Superior de Educação Física, Universidade Federal de Pelotas, Pelotas, RS, Brasil.

\author{
Gabriela Nilson ${ }^{1}$ \\ Eliane R Pardo ${ }^{1}$ \\ Luiz C. Rigo ${ }^{1}$ \\ Pedro C. Hallal ${ }^{1}$
}

\section{Resumo}

O presente estudo teve como objetivo analisar a autoimagem corporal de universitários, e foi realizado com estudantes do curso de bacharelado em Educação Física da Escola Superior de Educação Física da Universidade Federal de Pelotas (UFPEL). A pesquisa foi feita através da aplicação do BSQ-34 (Body Shape Questionaire) e do teste da Figura de Silhueta Corpórea (BFS - Body Figure Silhouettes). Os testes foram respondidos por 65 alunos, dos quais $63 \%$ eram homens. Pelo BSQ $81,5 \%$ dos alunos não apresentavam distorção da imagem corporal, $12 \%$ apresentavam leve distorção e 6\% apresentavam distorção moderada. Entre as mulheres, 16,7\% apresentavam distorção corporal moderada, enquanto que nenhum homem apresentou tal desfecho $(\mathrm{P}=0,005)$. Em relação aos resultados do $\mathrm{BFS}, 55,4 \%$ dos alunos estavam insatisfeitos com seus corpos, sendo que $35,4 \%$ deles gostariam de obter uma silhueta mais fina/magra e $20 \%$ de obter uma silhueta mais forte/maior. $\mathrm{Na}$ comparação por sexo, $22 \%$ dos homens e $58,3 \%$ das mulheres gostariam de ter uma silhueta mais magra/fina ( $\mathrm{P}=0,004)$. Além disso, 29,3\% dos homens gostariam de ter uma silhueta mais forte/maior. Boa parte dos acadêmicos do curso de bacharelado em Educação Física da UFPEL está insatisfeita com seus corpos, um fenômeno que não se restringe as mulheres. O corpo, a saúde e a estética corporal são demandas emergentes da sociedade que merecem receber uma atenção especial na formação dos profissionais de Educação Física.

\section{Palavras-chave}

Imagem corporal; Estética; Obesidade; Universidades.

\begin{abstract}
The aim of this study was to evaluate body self-image of university students. The study was conducted with students from the Physical Education School of the Federal University of Pelotas, Brazil (UFPEL). We used the Body Shape Questionnaire (BSQ-34) Body Figure Silhouettes (BFS). The sample comprised 65 students, of whom $63 \%$ were men. Based on BSO results, $81.5 \%$ of the students had no body image distortion, $12 \%$ presented light body image distortion and $6 \%$ presented moderate body image distortion. Among women, $16.7 \%$ presented moderate body image distortion, whereas this proportion was $0 \%$ among men $(P=0.005)$. In relation to BFS results, $55.4 \%$ of the students were unhappy with their bodies; $35.4 \%$ would like to be thinner and $20 \%$ would like to be stronger/bigger. In the sex comparisons, $22 \%$ of the men and $58.3 \%$ of the women would like to be thinner $(P=0.004)$. Furthermore, 29.3\% of the men would like to be stronger/bigger. A high proportion of the Physical Education School students from UFPEL is unhappy with their bodies, a phenomenon which is not restricted to women. Body, health and esthetics are emerging demands of contemporary societies that deserve further attention in undergraduate courses in Physical Education.
\end{abstract}

\section{Keywords}

Body image; Esthetics; Obesity; Universities. 


\section{INTRODUÇÃO}

A sociedade contemporânea convive com inúmeros discursos referentes à indústria da estética corporal. ${ }^{1,2}$ Os males contemporâneos do corpo, anorexia, vigorexia, obesidade, cada dia parecem estar mais presente e são preocupações constantes entre profissionais da área da saúde. ${ }^{3}$ Dois constructos que vêm sendo estudados na área são a distorção da imagem corporal e a satisfação com a imagem corporal. Estudo com estudantes de nutrição encontrou uma média correspondente à distorção leve de imagem corporal, sendo que 6,4\% dos alunos tinham distorção grave. ${ }^{4}$ Estudo com alunos de medicina encontrou distorção moderada da imagem corporal em 2,2\% dos homens e 9,9\% das mulheres. ${ }^{5}$ Existem evidências de que mulheres tendem a apresentar maior risco de comportamentos inadequados relacionados à distorção da imagem corporal quando comparadas aos homens ${ }^{6}$.

Estudos sobre a silhueta corpórea também têm trazido resultados interessantes. Em uma população de meninas adolescentes de Belo Horizonte, 67,2\% da amostra desejava ter um corpo mais magro. ${ }^{7}$ Entre universitárias da área da saúde de todo Brasil, a prevalência de insatisfação corporal foi de $64,4 \%$, sendo que a maioria dessas estudantes gostariam de emagrecer ${ }^{8}$. Estudo prévio no Sul do país mostrou que $43,3 \%$ dos homens sentem-se insatisfeitos por estarem mais magros do que gostariam, enquanto $62,4 \%$ das mulheres gostariam de emagrecer 9 .

Tais temas são extremamente relevantes para os profissionais de Educação Física $(\mathrm{EF})$, especialmente os bacharéis, que atuam diretamente com um público que costuma estar preocupado com padrões de corpo e de beleza. Assim, o presente estudo investigou, em uma amostra de estudantes do curso de Bacharelado em Educação Física, como os alunos lidam com a sua autoimagem corporal, se existe presença de distorções em suas percepções corporais e qual o grau de satisfação dos estudantes com seus corpos.

\section{MÉTODOS}

Foram selecionados todos os estudantes do primeiro e último anos do curso de bacharelado em Educação Física da Escola Superior de Educação Física (ESEF) da Universidade Federal de Pelotas (UFPEL). Otpou-se por selecionar estudantes no início e no final do curso para se eavaliar a existência ou não de diferenças oriundas pelo avançar do curso.

Foram utilizados dois instrumentos já amplamente utilizados em estudos que abordam esta temática: o Body Shape Questionaire - BSQ-34 ${ }^{10}$ e o teste de figuras de silhueta corpórea - $\mathrm{BFS}^{8}$. O BSQ-34 é uma escala Likert com 34 itens autopreenchíveis, construída para mensurar a preocupação com a forma corporal e com o peso. As perguntas possuem seis opções de respostas, e cada uma delas equivale a uma determinada pontuação: nunca ( 1 ponto), raramente ( 2 pontos), às vezes ( 3 pontos), frequentemente ( 4 pontos), muito frequentemente ( 5 pontos) e sempre (6 pontos). A partir das respostas faz-se a soma dos pontos obtidos em todo o teste e, assim, é possível classificar o nível de preocupação no que diz respeito à imagem corporal dos alunos em questão, da seguinte forma: $\leq 80$ pontos (ausência de distorção da imagem corporal); 81-110 pontos (distorção leve); 111-140 pontos (distorção moderada); $>140$ pontos (distorção grave). O BSQ apresenta bons índices de validade discriminante e confiabilidade ${ }^{4,5,8,10,12,13}$. Ele distingue também duas questões específicas relacionadas à imagem corporal: a estimativa do tamanho do corpo e os sentimentos em relação a ele, assim como a insatisfação ou desvalorização da forma física. 
Já o outro instrumento utilizado neste, o BFS, é comumente usado para avaliar distorções na imagem corporal de indivíduos, pois permite verificar as diferenças entre corpo atual e idealizado, além da imagem corporal no momento da aplicação do estudo. $\mathrm{O}$ instrumento foi validado em um estudo multiétnico a respeito de imagem corpora $^{11}$ e apresenta boa reprodutibilidade ${ }^{14,15}$. O BFS utilizado continha uma escala com oito figuras de silhuetas corporais, cada qual com um número correspondente. As silhuetas estavam dispostas em ordem crescente, da esquerda para a direita; a primeira figura ilustrava um corpo extremamente magro (número 1) e a última figura um corpo extremamente gordo (número 8). Os estudantes deveriam indicar sua percepção pessoal com relação à forma corporal atual e, a seguir, indicar sua percepção própria com relação à forma corporal desejada.

Os professores, contatados previamente para conhecimento do teor e objetivos da pesquisa, concordaram em disponibilizar parte do tempo de suas aulas para a aplicação do questionário diretamente na sala de aula. No dia da coleta dos dados, em um primeiro momento, foi realizada uma breve explicação a respeito da pesquisa. Após a explanação foi pedido aos alunos que sentassem o mais afastado possível dos colegas para que todos pudessem responder ao questionário com privacidade. Anexado ao questionário estava o termo de consentimento livre e esclarecido, onde constava a explicação mais detalhada no que diz respeito ao sigilo dos dados obtidos. Os alunos que não estavam na aula no momento da aplicação foram procurados em outro momento para responderem ao questionário. $\mathrm{O}$ índice de massa corporal (peso/altura ${ }^{5}$ ) foi calculado com base em informações autorreferidas de peso e altura.

Os dados foram analisados utilizando procedimentos de estatística descritiva (proporções, médias e desvios-padrão) e analítica (teste do qui-quadrado para comparações de proporções). Os dados foram digitados numa planilha do programa Excel e posteriormente transferidos para o pacote estatístico Stata, no qual as análises foram conduzidas.

O protocolo foi submetido e aprovado no Comitê de Ética em Pesquisa da Escola Superior de Educação Física da Universidade Federal de Pelotas.

\section{RESULTADOS}

Os testes BSQ-34 e BFS foram respondidos por 65 alunos, dos quais 63\% eram homens. Trinta e dois alunos eram ingressantes e 33 formandos. Apenas um aluno apresentou IMC $<18,5 \mathrm{~kg} / \mathrm{m}^{2}, 51$ alunos apresentaram IMC entre 18,5 e 24,9 kg/ $\mathrm{m}^{2}(78,5 \%)$ e 13 alunos tinham IMC $\geq 25 \mathrm{~kg} / \mathrm{m}^{2}$ (20\%). No total, $81,5 \%$ dos alunos não apresentavam distorção da imagem corporal, $12 \%$ apresentavam leve distorção e 6\% apresentavam distorção moderada. Não houve presença de distorção grave entre os alunos respondentes. A média do escore de distorção corporal foi de 61,4 pontos, $(\mathrm{DP}=23,1)$. Em relação aos resultados do $\mathrm{BFS}, 55,4 \%$ dos alunos estavam insatisfeitos com seus corpos, sendo que $35,4 \%$ deles gostariam de obter uma silhueta mais fina/magra e $20,0 \%$ de obter uma silhueta mais forte/maior. Para os escores de satisfação corporal a média obtida foi de 0,21 pontos $(\mathrm{DP}=$ $1,04)$, sendo que o valor zero tinha significado de satisfação completa do aluno com seu corpo (Tabela 1).

Realizamos também análises separadas de algumas questões entre as 34 que compõem o BSQ as quais chamam atenção para a elevada pontuação dos respondentes. As questões $02,04,09,12,22,30$ e 34 estão ligadas diretamente à autopercepção da forma do corpo. Na questão 02 , pouco mais de $52 \%$ dos alunos preo- 
Tabela 1 - Descrição da amostra de universitários de Educação Física.

\begin{tabular}{|c|c|c|}
\hline Variáveis categóricas & $\mathrm{N}$ & $\%$ \\
\hline \multicolumn{3}{|l|}{ Gênero } \\
\hline Masculino & 41 & 63,1 \\
\hline Feminino & 24 & 36,9 \\
\hline \multicolumn{3}{|l|}{ Estágios do curso } \\
\hline Ingressantes & 32 & 49,2 \\
\hline Formandos & 33 & 50,8 \\
\hline \multicolumn{3}{|l|}{ Índice de massa corporal } \\
\hline$<18,5 \mathrm{~kg} / \mathrm{m}^{2}$ & 1 & 1,5 \\
\hline $18,5-24,9 \mathrm{~kg} / \mathrm{m}^{2}$ & 51 & 78,5 \\
\hline$\geq 25,0 \mathrm{~kg} / \mathrm{m}^{2}$ & 13 & 20,0 \\
\hline \multicolumn{3}{|l|}{ Distorção da imagem corporal } \\
\hline Ausência de distorção & 53 & 81,5 \\
\hline Distorção leve & 8 & 12,3 \\
\hline Distorção moderada & 4 & 6,2 \\
\hline \multicolumn{3}{|l|}{ Satisfação corporal } \\
\hline Satisfeito & 29 & 44,6 \\
\hline Gostaria de ter uma silhueta mais magra & 23 & 35,4 \\
\hline Gostaria de ter uma silhueta mais forte & 13 & 20,0 \\
\hline \multicolumn{3}{|l|}{ Variáveis numéricas } \\
\hline & Média & DP \\
\hline Índice de massa corporal (kg/m²) & 23,0 & 2,5 \\
\hline Escore de distorção de imagem corporal* & 61,4 & 23,1 \\
\hline Escore de satisfação corporal ${ }^{\$}$ & 0,21 & 1,04 \\
\hline
\end{tabular}

*Quanto maior o escore, maior a distorção da imagem corporal.

\$O valor 0 indica satisfação completa com a silhueta. Valores positivos indicam preferência por uma silhueta mais fina/magra e valores negativos indicam preferência por uma silhueta mais forte/maior.

cupava-se com sua forma física a ponto de fazer dieta. Já a questão 04 demonstrou que 59\% dos alunos tinham receio de engordar.Já nas questões 09 e 12, 40\% e 44\% dos alunos, respectivamente, demonstraram preocupação com sua forma física ao comparar-se com pessoas do mesmo gênero. A questão 22 apontou que cerca de $30 \%$ dos alunos sentiam-se contentes ao sentir seu estômago vazio. Na questão 30, verificou-se pouco mais de 30\% dos alunos beliscava áreas do corpo para verificar o quanto havia de gordura nestes locais. Finalmente, a questão 34 demonstrou que $76 \%$ dos alunos preocupavam-se com sua forma física a ponto de sentir que deviam fazer exercícios (Tabela 2).

Os valores apresentados na tabela 3 são referentes ao IMC, distorção de imagem corporal (BSQ-34) e satisfação corporal (BFS - figura de silhueta corpórea) conforme gênero e estágios do curso. O percentual de sobrepeso/obesidade (IMC $\left.\geq 25 \mathrm{~kg} / \mathrm{m}^{2}\right)$ foi de $24,4 \%$ entre os homens e $12,5 \%$ entre as mulheres $(\mathrm{P}=0,24)$. Para a variável distorção da imagem corporal (BSQ-34), na comparação entre gêneros encontrou-se um valor estatisticamente significativo $(\mathrm{P}=0,005)$. Os valores apresentados pelos alunos estão distribuídos da seguinte forma: ausência de distorção: homens 92,7\% e mulheres 62,5\%; com leve distorção: homens 7,3\% e mulheres $20,8 \%$; com distorção moderada: homens $0 \%$ e mulheres $16,7 \%$. Na comparação para alunos ingressantes e formandos o valor $\mathrm{P}$ foi de 0,47 , indicando não haver associação significativa entre estágio do curso e distorção da imagem corporal. 
Tabela 2 - Descrição de alguns itens que compõem a escala de distorção da imagem corporal BSQ34 e da satisfação corporal (BFS) nos acadêmicos de Educação Física.

\begin{tabular}{lccc}
\hline QUESTÕES & $1^{*}$ & $2^{\#}$ & $3^{\&}$ \\
\hline $\begin{array}{l}\text { 2. Sua preocupação com sua forma física chega } \\
\text { ao ponto de você pensar que deveria fazer } \\
\text { uma dieta? }\end{array}$ & $47,6 \%$ & $32,3 \%$ & $20 \%$ \\
\hline $\begin{array}{l}\text { 4. Você tem receio de que poderia engordar ou } \\
\text { ficar mais gorda (o)? }\end{array}$ & $40 \%$ & $41,5 \%$ & $18,4 \%$ \\
\hline $\begin{array}{l}\text { 9. Estar com pessoas magras do mesmo sexo } \\
\text { que você faz você reparar em sua forma física? }\end{array}$ & $60 \%$ & $33,9 \%$ & $6,1 \%$ \\
\hline $\begin{array}{l}\text { 12. Você tem reparado na forma física de } \\
\text { outras pessoas do mesmo sexo que o seu, ao } \\
\text { se comparar, tem se sentido em desvantagem? }\end{array}$ & $55,3 \%$ & $40 \%$ & $4,6 \%$ \\
\hline $\begin{array}{l}\text { 22. Você se sente mais contente em relação } \\
\text { à sua forma física quando seu estômago está } \\
\text { vazio (por exemplo, pela manhã)? }\end{array}$ & $70,7 \%$ & $24,6 \%$ & $6,1 \%$ \\
\hline $\begin{array}{l}\text { 30. Você belisca áreas de seu corpo para ver o } \\
\text { quanto há de gordura? }\end{array}$ & $64,6 \%$ & $29,2 \%$ & $6,1 \%$ \\
\hline $\begin{array}{l}\text { 34. A preocupação com sua forma física leva } \\
\text { você a sentir que deveria fazer exercícios? }\end{array}$ & $23 \%$ & $47,6 \%$ & $29,2 \%$ \\
\hline
\end{tabular}

* Referente a respostas 1 e 2 de cada questão ( $1=$ Nunca; $2=$ Raramente)

\# Referente a respostas 3 e 4 de cada questão ( $3=$ Às vezes; $4=$ Frequentemente)

$\&$ Referente a respostas 5 e 6 de cada questão ( $5=$ Muito frequentemente; $6=$ Sempre)

Tabela 3 - Índice de massa corporal, distorção de imagem corporal e satisfação corporal conforme gênero e estágio do curso.

\begin{tabular}{|c|c|c|c|c|c|c|}
\hline \multirow{2}{*}{ Variáveis } & Homens & Mulheres & Valor $\mathrm{P}^{*}$ & Ingressantes & Formandos & Valor $\mathrm{P}^{*}$ \\
\hline & $\%$ & $\%$ & & $\%$ & $\%$ & \\
\hline Índice de massa corporal & & & 0,24 & & & 0,40 \\
\hline$<18,5 \mathrm{~kg} / \mathrm{m}^{2}$ & 0,0 & 4,2 & & 0,0 & 3,0 & \\
\hline $18,5-24,9 \mathrm{~kg} / \mathrm{m}^{2}$ & 75,6 & 86,3 & & 84,4 & 72,7 & \\
\hline$\geq 25,0 \mathrm{~kg} / \mathrm{m}^{2}$ & 24,4 & 12,5 & & 15,6 & 24,2 & \\
\hline Distorção da imagem corporal & & & 0,005 & & & 0,47 \\
\hline Ausência de distorção & 92,7 & 62,5 & & 81,3 & 81,8 & \\
\hline Distorção leve & 7,3 & 20,8 & & 15,6 & 9,1 & \\
\hline Distorção moderada & 0,0 & 16,7 & & 3,1 & 9,1 & \\
\hline Satisfação corporal & & & 0,004 & & & 0,68 \\
\hline Satisfeito & 48,8 & 37,5 & & 40,6 & 48,5 & \\
\hline Gostaria de ter uma silhueta mais magra & 22,0 & 58,3 & & 40,6 & 30,3 & \\
\hline \multirow[t]{2}{*}{ Gostaria de ter uma silhueta mais forte } & 29,3 & 4,2 & & 18,8 & 21,1 & \\
\hline & Média (DP) & Média (DP) & Valor P & Média (DP) & Média (DP) & Valor $\mathrm{P}$ \\
\hline Índice de massa corporal $\left(\mathrm{kg} / \mathrm{m}^{2}\right)$ & $23,5(2,4)$ & $22,1(2,6)$ & 0,03 & $23,1(2,4)$ & $22,9(2,7)$ & 0,81 \\
\hline Escore de distorção de imagem corporal\& & $53,0(15,9)$ & $76,7(26,7)$ & $<0,001$ & $60,5(22,7)$ & $62,2(23,9)$ & 0,78 \\
\hline Escore de satisfação corporal\$ & $-0,12(0,93)$ & $0,79(0,98)$ & $<0,001$ & $0,31(1,26)$ & $0,12(0,78)$ & 0,46 \\
\hline
\end{tabular}

* Teste do qui-quadrado

\&Quanto maior o escore, maior a distorção da imagem corporal.

$\$ O$ valor 0 indica satisfação completa com a silhueta. Valores positivos indicam preferência por uma silhueta mais fina/magra e valores negativos indicam preferência por uma silhueta mais forte/maior.

Para a variável de satisfação corporal (BFS), obtivemos um valor $\mathrm{P}=0,004$ para comparação entre gêneros; $48,8 \%$ dos homens e $37,5 \%$ das mulheres estavam satisfeitos com seus corpos; $22 \%$ dos homens e $58,3 \%$ das mulheres gostariam de ter uma silhueta mais magra/fina e 29,3\% dos homens e 4,2\% das mulheres gostariam 
de ter uma silhueta mais forte/maior. Na comparação entre ingressantes e formandos o valor $\mathrm{P}$ foi de 0,68 , indicando ausência de associação entre estágio no curso e satisfação com o corpo.

Para a pontuação média de escore de distorção corporal na comparação entre gêneros observou-se um valor $\mathrm{P}<0,001$. Os resultados obtidos mostraram 53 pontos de média dos homens $(\mathrm{DP}=15,9)$ e 76,7 pontos de média das mulheres, $(\mathrm{DP}=26,7)$. No que diz respeito aos escores de satisfação corporal (BFS), a comparação entre gêneros apresentou um valor $\mathrm{P}<0,001$. A pontuação média dos homens foi de $-0,12$ pontos $(\mathrm{DP}=0,93)$ e a das mulheres foi 0,79 pontos $(\mathrm{DP}=0,98)$.

Em destaque também as questões sobre satisfação corporal (BFS), de acordo com a escala de silhuetas apresentada para os alunos. Os resultados mostraram que $13 \%$ das alunas percebiam seus corpos nas imagens 1 e 2 da tabela de silhuetas, $29 \%$ percebiam-se pela imagem 3 , cerca de $54 \%$ das alunas percebiam seus corpos pelas imagens 4 e 5 , e $4 \%$ pela imagem 6 (Figura1). Já para a questão seguinte (corpo ideal), $25 \%$ das alunas gostariam de parecer como a imagem $2,50 \%$ gostariam de ser como a imagem 3, e outros $25 \%$ das alunas, de parecer como a imagem 4 .

O seu corpo se parece com qual das imagens?

-Imagem 3 -Imagem 4 -Imagem 5 -Imagem 6
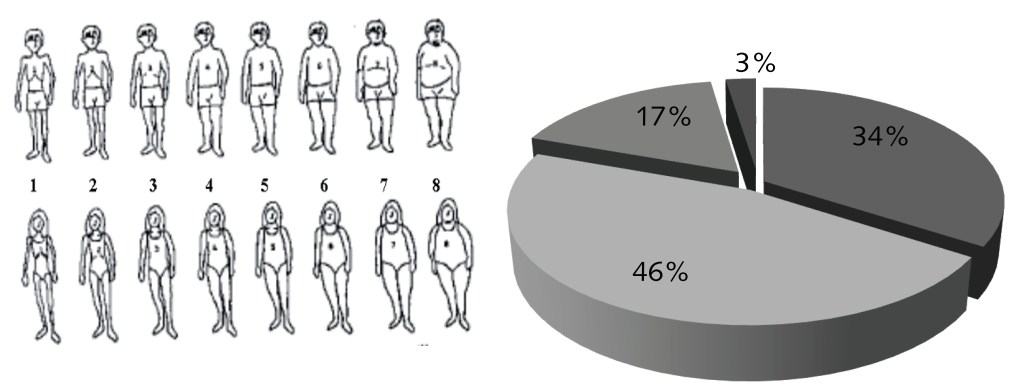

Qual destas imagens mostra como você gostaria de se parecer?

-Imagem 2 Imagem 3 -Imagem 4 Imagem 5 Imagem 6

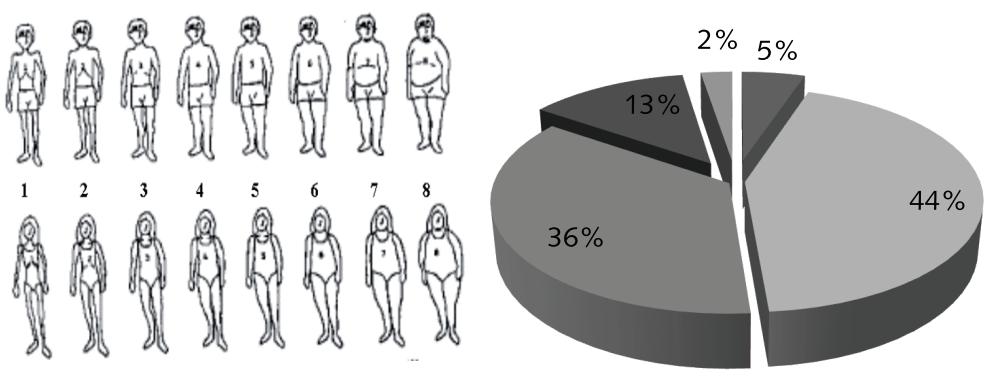

Figura 1 - Comparação entre a percepção da imagem corporal e o ideal entre as mulheres.

No caso dos homens (Figura 2) verificamos que 34\% dos alunos percebiam seus corpos na imagem 3, 46\% percebiam seus corpos na imagem 4, 17\% na imagem 5 e $3 \%$ na imagem 6 . Em relação ao corpo ideal, $5 \%$ gostariam de parecer com a imagem $2,44 \%$ com a imagem $3,36 \%$ com a imagem $4,13 \%$ com a imagem 5 e $2 \%$ com a imagem 6. 
O seu corpo se parece com qual das imagens?

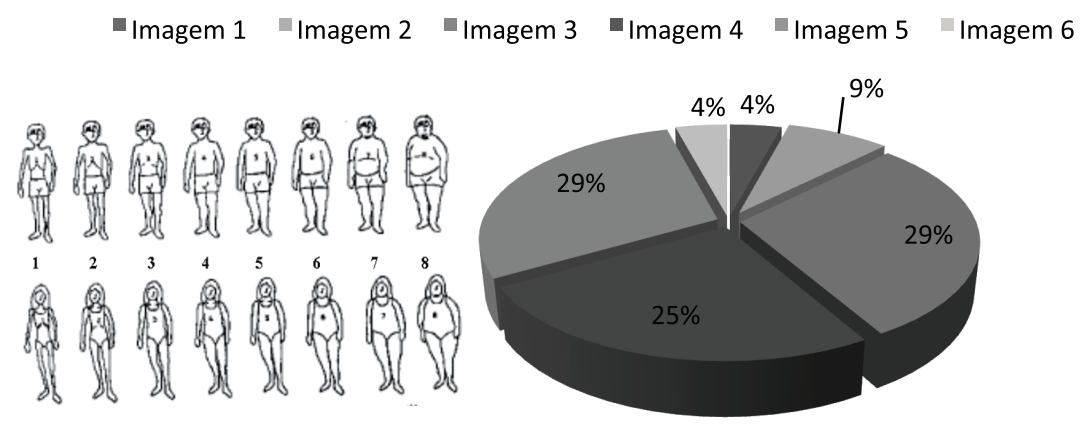

Qual destas imagens mostra como você gostaria de se parecer?

-Imagem 2 Imagem 3 Imagem 4

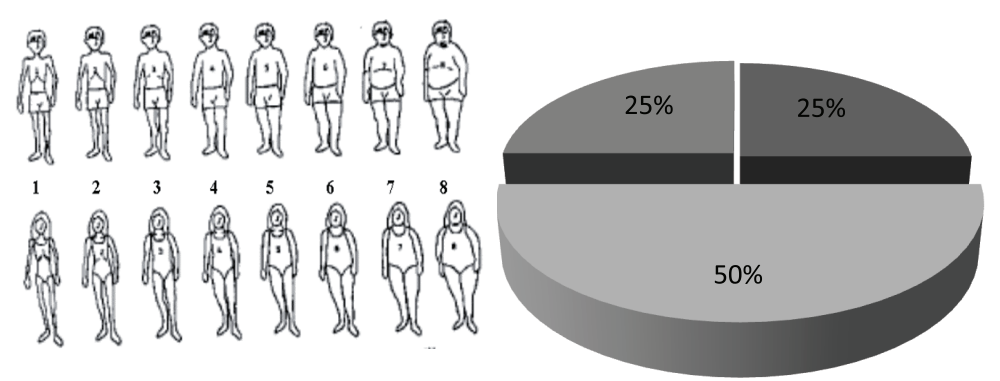

Figura 2 - Comparação entre a percepção da imagem corporal e o ideal entre os homens.

\section{DISCUSSÃO}

Os resultados apresentados indicam que 18,5\% dos alunos participantes apresentaram algum tipo de distorção, distribuída entre leve e moderada, sendo a distorção da imagem corporal mais comum entre as mulheres. No estudo de Bosi et $\mathrm{al}^{4}$, com estudantes de Nutrição, a prevalência entre as mulheres foi próxima de 30\%, valor semelhante ao encontrado em nosso estudo para as mulheres (37,5\%). O escore médio obtido no estudo de Bosi et al. ${ }^{4}$ foi de 81,2 pontos, enquanto em nosso estudo tal valor foi de 76,7 pontos para as mulheres e 53,0 para os homens. Já no estudo de Di Pietro \& Silveira ${ }^{5}$, em uma população de estudantes de medicina, o escore médio para homens foi 58,7 pontos, parecido com os resultados apresentados neste estudo.

Para a variável de satisfação corporal (BFS) o resultado do presente estudo mostrou que há índice de 55,4\% de insatisfação com a forma do corpo entre os estudantes que participaram da pesquisa; números parecidos foram encontrados em pesquisas com populações universitárias ${ }^{16,17}$. Entre os homens, a maioria quer uma silhueta mais forte, enquanto entre as mulheres a maioria quer uma silhueta mais magra.

Algumas limitações do estudo precisam ser consideradas. Embora amplamente utilizado, o IMC calculado com base em peso e altura autorreferidos pode ser um fator limitante deste estudo. Por se tratar de uma população de estudantes universitários do curso de Educação Física, os resultados não podem ser imediatamente extrapolados para a população em geral.

Estudo realizado com uma população de adolescentes do gênero feminino, em Belo Horizonte, apontou que $67,2 \%$ da amostra desejava ter um corpo mais magro $^{7}$. Em pesquisa semelhante, com a utilização somente do BFS em estudantes 
universitárias da área da saúde de todo Brasil, Alvarenga et al. ${ }^{8}$ encontraram um total nacional de $64,4 \%$ de insatisfação corporal. Quadros et al. ${ }^{9}$ estudaram universitários do Sul do Brasil, mostrando que 43,3\% dos homens sentem-se insatisfeitos por estarem mais magros do que gostariam e $62,4 \%$ das mulheres gostariam de emagrecer.

A insatisfação com o corpo pode influenciar de forma pontual a prática de dietas e outras estratégias restritivas de alimento. Portanto, deve-se atentar para a importância da prevenção a fim de se evitar casos de transtornos do comportamento alimentar, principalmente em alunos universitários. Legnani, et al. ${ }^{18}$ ao investigar as associações entre transtornos alimentares e distorção de imagem corporal em universitários de Educação Física, concluiu que os estudantes que tinham distorções de imagem corporal apresentavam uma chance 5,3 vezes maior de também apresentarem transtornos alimentares. Há estudos que apontam que profissionais que atuam em áreas onde há forte relação com o corpo, saúde e ou estética corporal, compõem um grupo bastante vulnerável ao desenvolvimento desses transtor$\operatorname{nos}^{4,19,20}$.

Os resultados alcançados nesse estudo mostram que há uma necessidade de os temas relacionados à imagem e a autoimagem corporal receberem uma maior atenção nos currículos superiores de Educação Física, conclusão similar a que assinalaram Da Silva et. al. ${ }^{20}$ Especialmente pelo fato de o profissional de Educação Física intervir na construção da imagem corporal de outros indivíduos é fundamental que ele tenha uma formação que o possibilita analisar e problematizar os discursos presentes no campo ${ }^{21}$ midiático, que tratam do corpo, da imagem e da autoimagem corporal. Principalmente, os efeitos que podem decorrer de discursos que insistem em instituir padrões de corpo para as mulheres e para os homens. ${ }^{1,2}$

Outra contribuição do estudo foi o fato dele ter investigado acadêmicos de Educação Física de ambos os sexos, aspecto não considerado por alguns estudos que trouxeram importantes contribuições sobre esse tema, mas restringiram-se ao sexo feminino. ${ }^{22}$. Esse ponto merece ser salientado, pois dificilmente os problemas do corpo moderno são exclusivo de um gênero. ${ }^{1,2}$ Butler, ${ }^{23}$ por exemplo, problematiza a própria existência de dois gênero (um masculino e outro feminino), e argumenta que é importante superar essa classificação dicotômica, pois ela representa o resultado de uma construção histórica cultural que esteve referendada prioritariamente nas diferenças biológicas entre homens e mulheres.

\section{Contribuições dos autores}

GN idealizou o estudo, conduziu a revisão da literatura e liderou a escrita do artigo. EP supervisionou o trabalho em todas as etapas. LCR participou do planejamento do estudo e auxiliou na redação do manuscrito. PH conduziu a análise dos dados. Todos os autores revisaram versões prévias desse artigo e aprovaram a versão submetida.

\section{REFERÊNCIAS}

1. Sant'anna DB. Corpos de passagem: ensaios sobre a subjetividade contemporânea. São Paulo: Estação Liberdade, 2001.

2. Miskolci R. Corpos elétricos: do assujeitamento à estética da existência. Estudos Feministas. 2006; 14: 681-693.

3. Alvarenga MS. Bulimia Nervosa: avaliação do padrão e comportamento alimentares. 2001.226f. Tese (Doutorado) - Faculdade de Ciências Farmacêuticas da USP. Faculdade de Economia, Administração e Contabilidade da USP. Faculdade de Saúde Pública da USP - Curso de Pós- 
graduação Interunidades em Nutrição humana Aplicada. Universidade de São Paulo.

4. Bosi MLM, Luiz RR, Morgado CM, Costa ML, Carvalho RJ. Autopercepção da imagem corporal entre estudantes de nutrição: um estudo no município do Rio de Janeiro. J Bras Psiquiatr 2006;55:108-13.

5. Di Pietro M, Silveira DX. Internal validity, dimensionality and performance of the Body Shape Questionnaire in a group of Brazilian college students. Rev Bras Psiquiatr 2009; 31:21-4.

6. Saikali CJ, Soubhia CS, Scalfaro BM, Cordás TA. Imagem corporal nos transtornos alimentares. Rev Psiq Clín 2004;31:164-6.

7. Beling MTC. A autoimagem corporal e o comportamento alimentar de adolescentes do sexo feminino em Belo Horizonte, MG. 2008. 165f. Dissertação (Mestrado) - Faculdade de Medicina - Programa de Pós- graduação em Ciências da Saúde. Universidade Federal de Minas Gerais.

8. Alvarenga MS, Philippi ST, Lourenço BH, Sato PM, Scagliusi FB. Insatisfação com a imagem corporal em universitárias brasileiras. J Bras Psiq 2010; 59:44-51.

9. Quadros TMB, Gordia AP, Martins CR, Silva DAS, Ferrari EP, Petroski EL. Imagem corporal em universitários: associação com estado nutricional e sexo. Motriz 2010; 16:78-85.

10. Cooper P, Taylor MJ, Cooper Z, Fairburn CG. The Development and Validation of the Body Shape Questionnaire. Int J Eating Disord 1987;6:485-94.

11. Stunkard A, Sorenson T, Schulsinger F. Use of the Danish Adoption Register for the study of obesity and thinness. In S. Kety, L.P. Rowland, R.L. Sidman, \& S.W. Matthysse (Eds.), The Genetics of Neurological and Psychiatric Disorders (pp. 115-120). New York: Raven Press, 1983.

12. Oliveira FP, Bosi MLM, Vigário PS, Vieira RS. Comportamento alimentar e imagem corporal em atletas. Rev Bras Med Esporte 2003;9:348-56.

13. Russo R. Imagem corporal: construção através da cultura do belo. Movimento \& Percepção 2005;5:80-90.

14. Thompson JK, Gray J. Development and validation of a new body-image assessment scale. J Person Assess 1995;64:258-69.

15. Fernandes AER. Avaliação da imagem corporal, hábitos de vida e alimentares em crianças e adolescentes de escolas públicas e particulares de Belo Horizonte. 2007. Dissertação (Mestrado) - Faculdade de Medicina. Universidade Federal de Minas Gerais.

16. Coqueiro RS, Petroski EL, Pelegrini A, Barbosa AR. Insatisfação com a imagem corporal: avaliação comparativa da associação com estado nutricional em universitários. Revista de Psiquiatria do Rio Grande do Sul 2008;30:31-38.

17. Gonçalves TD, Barbosa MP, Rosa LCL, Rodrigues AM. Comportamento anoréxico e percepção corporal em universitários. J Bras Psiq 2008;57:166-70.

18. Legnani RFS, Leganani E, Pereira ÉF, et. al. Transtornos alimentares e imagem corporal em acadêmicos de Educação Física. Motriz 2012; 18: 84-91.

19. Bosi MLM, Luiz RR; Uchimura KY, Oliveira, FP. Comportamento Alimentar e Imagem Corporal entre estudantes de educação física. J Bras Psiq 2008; 57: 28-33.

20. Da Silva TR, Saenger G, Pereira ÉF. Fatores associados à imagem corporal em estudantes de Educação Física. Motriz, 2011; 17: 630 - 639.

21. Bourdieu P. Campo de Poder, Campo Intelectual: itinerario de un concepto. Buenos Aires, Montressor, 2002.

22. Garcia L, Milagres OG, Mourão L, Assis M, Palma A. Auto-percepção da imagem corporal em estudantes de educação física e medicina. Rev. Bras de Atividade Física \& Saúde 2011; 16: $25-30$.

23. Butler J. Problemas de gênero: feminismo e subversão da identidade. Rio de Janeiro, Civilização Brasileira, 2003. 